5. Okada, H., et al. 1998. Identification of a novel cisacting element for fibroblast-specific transcription of the FSP1 gene. Am. J. Physiol. 275:F306-F314.

6. Venkov, C.D., et al. 2007. A proximal activator of transcription in epithelial-mesenchymal transition. J. Clin. Invest. 117:482-491. doi:10.1172/JCI29544.

7. Kamada, S., and Miwa, T. 1992. A protein binding to CArG box motifs and to single-stranded DNA functions as a transcriptional repressor. Gene. 119:229-236

8. Bemark, M., Olsson, H., Heinegard, D., and Lean- derson, T. 1998. Purification and characterization of a protein binding to the SP6 kappa promoter. A potential role for CArG-box binding factor-A in kappa transcription. J. Biol. Chem. 273:18881-18890.

9. Mikheev, A.M., Mikheev, S.A., Zhang, Y., Aebersold, R., and Zarbl, H. 2000. CArG binding factor A (CBF-A) is involved in transcriptional regulation of the rat Ha-ras promoter. Nucleic Acids Res. 28:3762-3770

10. Friedman, J.R., et al. 1996. KAP-1, a novel corepressor for the highly conserved KRAB repression domain. Genes Dev. 10:2067-2078.

11. Schultz, D.C., Friedman, J.R., and Rauscher, F.J., 3rd. 2001. Targeting histone deacetylase complexes via $\mathrm{KRAB}$-zinc finger proteins: the PHD and bromodomains of KAP-1 form a cooperative unit that recruits a novel isoform of the Mi-2alpha subunit of NuRD. Genes Dev. 15:428-443.

12. Zeisberg, M., et al. 2003. BMP-7 counteracts TGFbeta1-induced epithelial-to-mesenchymal transition and reverses chronic renal injury. Nat. Med. 9:964-968.

\title{
To ablate or not to ablate? HSCs in the T cell driver's seat
}

\author{
Claudio Anasetti and James J. Mulé
}

H. Lee Moffitt Comprehensive Cancer Center, Tampa, Florida, USA.

\begin{abstract}
The combination of the induction of lymphopenia and vaccination and/or $T$ cell transfer is garnering much attention for cancer treatment. Preclinical studies have shown that the induction of lymphopenia by chemotherapy or radiation can enhance the antitumor efficacy of several distinct, cell-based immunotherapeutic approaches. The mechanism(s) by which such enhancement is achieved are being intensively studied, yet there is much opportunity for improvement. The animal studies reported by Wrzesinski and colleagues in this issue of the JCI are a promising and timely step in this direction (see the related article beginning on page 492). The authors have evaluated both the effect of increasing the intensity of lymphodepletion and the influence of HSC transfer on the in vivo function of adoptively transferred $\mathrm{CDB}^{+}$ $T$ cells. We discuss their results in light of the evolving field and their implications for advancing cell-based immunotherapies for cancer.
\end{abstract}

Current immunization approaches attempt to activate and expand the tumor-reactive $\mathrm{T}$ cell population in hosts with an intact immune system. There is much evidence that within the immune system of cancer patients, tumor-induced suppression and immune-based regulatory factors are present that may limit the effectiveness of vaccine-induced, tumor-specific $\mathrm{T}$ cells (1). An alternative approach is to induce lymphopenia (a reduction in lymphocyte number) in hosts, allowing residual host or transferred naive or antigen-specific donor $\mathrm{T}$ cells to undergo homeostasis-driven proliferation to restore the memory $\mathrm{T}$ cell compartment. Several potential advantages are offered by this strategy. For example, in

Nonstandard abbreviations used: BMT, bone marrow transplantation; MART-1, melanoma-associated antigen recognized by $\mathrm{T}$ cells 1 ; TBI, total body irradiation; $\mathrm{T}_{\mathrm{cm}}$ cell, central memory $\mathrm{T}$ cell; $\mathrm{T}_{\mathrm{em}}$ cell, effector memory T cell; TIL, tumor-infiltrating lymphocyte.

Conflict of interest: The authors have declared that no conflict of interest exists.

Citation for this article: J. Clin. Invest. 117:306-310 (2007). doi:10.1172/JCI30973. addition to eliminating inhibitory immune cells in the host such as Tregs, lymphomyeloid reconstitution may overcome inherent defects in $\mathrm{T}$ cell signaling and may strengthen the costimulatory functions of APCs (2). Induction of lymphopenia can lead to an increased production and availability of immune response-stimulating cytokines such as IL-7 and IL-15, resulting in enhanced $\mathrm{CD} 8^{+} \mathrm{T}$ cell activity $(3,4)$. Other studies have shown enhanced $\mathrm{T}$ cell trafficking into tumors after induction of lymphopenia $(5,6)$, as well as enhanced intratumoral proliferation of effector cells (7). It is postulated that vaccination during homeostasis-driven proliferation may serve to educate the developing $\mathrm{T}$ cell repertoire and lead to enhanced $T$ cell memory against tumor-associated self antigens $(8,9)$.

Common methods to induce lymphopenia include treatment with low-dose total body irradiation (TBI) that produces mild, reversible myelosuppression (hence nonmyeloablative) or treatment with chemotherapeutic drugs such as cyclophosphamide (Cy) alone or in combination with fludara- bine, which can induce short-term and longer-term lymphopenia in mice and humans, respectively (10). Cy-induced lymphopenia can also enhance the induction of tumorspecific $C D 8^{+} \mathrm{T}$ cells and can lead to protective immunity against tumors $(11,12)$.

In this issue of the JCI, Wrzesinski and colleagues (13) report on a series of animal studies undertaken to determine whether it is possible to augment adoptively transferred $\mathrm{T}$ cell-mediated tumor destruction by increasing the intensity of lymphodepletion. The work moves the immunotherapy field forward by demonstrating, for the first time to our knowledge, a positive influence of HSC transfer on the in vivo function of adoptively transferred $\mathrm{CD} 8^{+} \mathrm{T}$ cells.

\section{Immunotherapy in the setting of nonmyeloablative lymphodepletion}

Adoptive transfer of naive or activated antigen-specific $\mathrm{T}$ cells immediately after induction of lymphopenia has been successful in inducing tumor regression in several murine models as well as in human clinical trials. In murine tumor models, induction of lymphopenia followed by adoptive transfer of peptide-specific $T$ cells alone led to regression of established tumors (8). Active vaccination in combination with lymphodepletion and adoptive $T$ cell transfer may further enhance immunity $(14,15)$. Studies have shown that active vaccination can skew the $T$ cell repertoire toward self or tumor-associated antigens during homeostatic proliferation $(16,17)$. Hu et al. $(18)$ have reported that tumor-specific $\mathrm{T}$ cells can preferentially expand in tumor vaccine-draining lymph nodes following adoptive transfer of naive 
T cells and injection of a melanoma vaccine in RAG1-deficient mice. DC-based vaccines are particularly attractive as a treatment adjunct, as homeostasis-driven proliferation has been shown to be dependent on interactions of $\mathrm{T}$ cells with self peptide MHC on DCs (19), and such vaccines can boost the antitumor activity of adoptively transferred, tumor antigen peptide-specific T cells in vivo (20). Lymphodepleting chemotherapy in combination with adoptive transfer of naive T cells and DC-based immunotherapy can lead to rejection of established melanoma (14). The most effective antitumor immunity is induced when vaccination and reconstitution are performed concomitantly, as delayed vaccination may result in T cells with less antitumor potency (14).
In the mouse, lymphopenia drives not only the proliferation of CD $44{ }^{\text {hi }} \mathrm{CD} 62$ ligand ${ }^{\text {lo }}\left(\mathrm{CD} 44^{\text {hi }}{ }^{\mathrm{CD}} 62^{\mathrm{L}}{ }^{\text {lo }}\right)$ effector memory $\mathrm{T}\left(\mathrm{T}_{\mathrm{em}}\right)$ cells, but also induces naive $\mathrm{T}$ cells undergoing homeostasis-driven proliferation to convert to a memory or activated state (21-23). Both $\mathrm{T}_{\mathrm{em}}$ cells and "central" memory $\mathrm{T}\left(\mathrm{T}_{\mathrm{cm}}\right)$ cells, expressing high levels of CD62L and C-C motif chemokine receptor 7 (CCR7), have been shown to be important for long-lasting immune responses (24, $25)$. While $\mathrm{T}$ cells are phenotypically $\mathrm{T}_{\mathrm{em}}$ cells during homeostatic proliferation, DCbased vaccines can accelerate the development of $\mathrm{T}_{\mathrm{cm}}$ cells (26). $\mathrm{T}_{\mathrm{cm}}$ cells proliferate better in response to antigenic stimulation, leading to enhanced protection against antigenic challenge (27). A recent study demonstrated in melanoma-bearing mice that cytokine therapy in combination with adoptive $T$ cell transfer and a DC-based vaccine in the setting of lymphopenia can lead to a higher number of $\mathrm{T}_{\mathrm{cm}}$ cells, which correlated with long-term survival (28).

In cancer patients, nonmyeloablative induction of lymphopenia enhanced the efficacy of adoptively transferred, tumor antigen-specific T cells (29). Transfer of melanoma-associated antigen recognized by $\mathrm{T}$ cells 1 -specific (MART-1-specific), autologous tumor-infiltrating lymphocytes (TILs) and high-dose IL-2 therapy after chemotherapy-induced lymphopenia resulted in the rapid expansion in vivo of a clonal population of $\mathrm{T}$ cells specific for the MART-1 antigen and resulted in the destruction of metastatic tumors and induction of autoimmunity against nor-

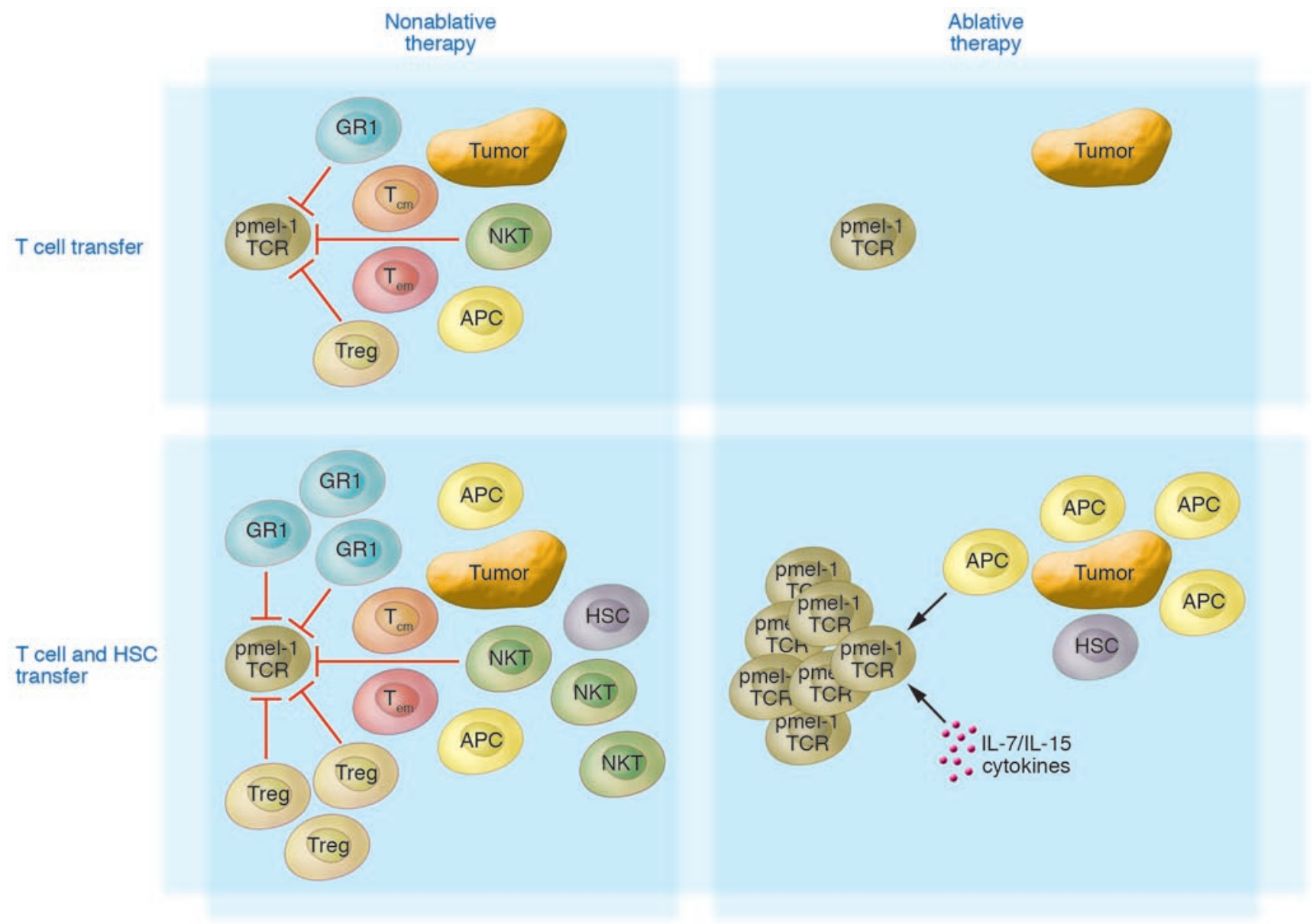

Figure 1

Schematic of the promotion of expansion and function of adoptively transferred antitumor CD8 ${ }^{+} \mathrm{T}$ cells following myeloablation and HSC rescue. HSC transplant, given as part of the myeloablative regimen, can significantly augment the expansion and the antitumor impact of adoptively transferred self/tumor antigen-reactive T cells. Myeloablation effectively removes host inhibitory cells, opens up available space (the so-called Lebensraum effect), and destroys cells serving as a cytokine sink. The operative mechanism by which HSCs positively impact the transferred T cells is currently unknown but could include the production of APCs and T cell homeostatic cytokines (e.g., IL-7 and IL-12). GR1, suppressive monocytes; HSC, lin-c-kit ${ }^{+}$HSC; pmel-1 TCR, gp100 melanoma-associated antigen-specific T cell; Treg, CD4 ${ }^{+}$CD $25^{+}$Foxp3 ${ }^{+}$Tregs; tumor, melanoma expressing gp100. 
mal tissues expressing the MART-1 antigen (29-31). In some of these patients, regression and resolution of large tumors and disseminated disease were observed.

\section{Immunotherapy in the setting of myeloablative lymphodepletion}

In mice recovering from lymphopenia following myeloablative doses of TBI and transplantation of whole bone marrow (i.e., containing resident $\mathrm{T}$ cells) (BMT), DCbased vaccines can expedite $T$ cell reconstitution and activation $(32,33)$. DC-based vaccination following myeloablative TBI and whole BMT is an effective means to induce regression or growth retardation of established tumors in murine syngeneic (34) and allogeneic models (35), respectively.

It is possible that resident $T$ cells contained in the bone marrow from a naive donor may be more reactive to vaccination with self antigens than the endogenous $\mathrm{T}$ cells remaining after nonmyeloablative chemotherapy in a tumor-bearing host. Indeed, homeostasis-driven proliferation restores only the memory $\mathrm{T}$ cell compartment, whereas thymopoiesis is required to reconstitute the naive $\mathrm{T}$ cell compartment (36). After whole BMT, both homeostatic proliferation of resident $\mathrm{T}$ cells in the bone marrow and thymopoiesis together lead to reconstitution of the $\mathrm{T}$ cell compartment. In addition, bone marrow contains a high percentage of $\mathrm{T}_{\mathrm{cm}}$ cells (37). Whereas induction of $\mathrm{T}_{\mathrm{em}}$ cells is important for a peripheral immune response, $\mathrm{T}_{\mathrm{cm}}$ cells are superior in trafficking to peripheral lymph nodes and inducing strong systemic immunity (27). Transfer of purified T cells from the bone marrow of tumor lysate-pulsed DC-vaccinated mice could lead to regression of breast cancer and melanoma (32). In another study, adoptive transfer of $\mathrm{T}_{\mathrm{cm}}$ cells was more effective than transfer of $\mathrm{T}_{\mathrm{em}}$ cells at inducing tumor regression in a murine model of melanoma (24), indicating the importance of $\mathrm{CD}^{+} \mathrm{T}_{\mathrm{cm}}$ cells for the induction of strong, systemic antitumor immunity.

In the setting of lymphopenia after HSC transplantation, adoptive $\mathrm{T}$ cell immunotherapy as a prophylactic or strategy for treating CMV, EBV, and adenovirus infections after transplantation has been conducted with promising outcomes (38-40). Strategies for employing $T$ cells specific for minor histocompatibility antigens ( $\mathrm{mHA}$ ) to augment the graft-versus-leukemia effect that contributes to tumor eradication are also being employed $(41,42)$.
In other clinical studies, strategies have included transfer of HSCs from a donor immunized with tumor antigen (idiotype proteins) to an allogeneic HSC recipient to avoid relapse in the setting of multiple myeloma (43, 44). Moreover, combination immunotherapy consisting of a single early posttransplantation infusion of in vivo vaccine-primed and ex vivo costimulated autologous $\mathrm{T}$ cells followed by posttransplantation booster immunizations can improve the severe immunodeficiency associated with high-dose chemotherapy and can lead to the induction of clinically relevant immunity in adults within a month after transplantation (45). In cases where the functional quality of $\mathrm{T}$ cells for adoptive transfer against tumors or microbial/viral infections is compromised, rescue can be achieved by the use of IL-15 (46), anti-CD40 (47), or program death 1 (PD-1) engagement blockade (48), potentially allowing for a broader use of adoptive immunotherapy in these settings.

\section{HSCs appear to be the key}

The study by Wrzesinski et al. reported in this issue of the JCI shows that prior treatment with a myeloablative TBI dose requiring an HSC transplant can enhance both the expansion of the adoptively transferred, tumor-reactive $\mathrm{T}$ cell population and the efficacy of tumor treatment in mice as compared with a nonmyeloablative TBI dose not requiring an HSC transplant (13). Importantly, the mere reduction of the levels of regulatory host elements and host cells competing for cytokines (socalled cytokine sinks) was not sufficient to increase $\mathrm{T}$ cell proliferation. Transplantation of purified HSCs drove the expansion of both transferred effector $\mathrm{T}$ cells and cells that had survived lymphodepletion, which correlated with elevated serum levels of both IL-7 and IL-15 (Figure 1). HSCdriven $\mathrm{T}$ cell expansion was also observed in nonmyeloablated mice that also received an HSC transplant. However, it was only profound lymphodepletion, by radiation-induced myeloablation or by genetic means, that preferentially boosted the expansion of the transferred tumor-specific $\mathrm{T}$ cells, resulting in successful tumor treatment. Moreover, transferred T cells did not require in vitro preactivation to achieve successful treatment in the myeloablated setting. However, TCR engagement of naive $T$ cells was a requirement, because HSC-driven expansion of naive $T$ cells was observed in WT mice and not in mice lack- ing $\beta_{2}$-microglobulin $\left(\beta_{2} \mathrm{~m}^{-/-}\right.$mice, which are virtually devoid of functional MHC class I). This was not the case for effector cells, the HSC-driven expansion of which required neither MHC class I molecules on host cells nor additional vaccination. Last, surviving host cells negatively influenced the tumor treatment capacity of transferred $\mathrm{CD}^{+} \mathrm{T}$ cells even when they were present in small numbers after TBI-mediated lymphodepletion. It is noteworthy that these new findings could probably not have been uncovered without a very intricate mouse model. The authors employed their highly sensitive pmel-1 TCR-transgenic mouse model, in which $\mathrm{CD}^{+} \mathrm{T}$ cells recognize the gp100 tumor antigen, which is also relevant as a target in human melanoma clinical trials (49).

The current study raises a few key points for further consideration. First, conditioning with irradiation per se has at least two opposing activities on tumor immunity: (a) It depletes, in a dose-dependent manner, resident hematopoietic cells, including APCs (50), thus impairing antigen-specific and costimulation-driven expansion of tumorspecific $T$ cells. The role of resident APCs in the stimulation of transferred, tumor-reactive T cells after allogeneic HSC transplantation has been shown by others $(51,52)$. (b) It depletes Tregs, NKT cells, and Gr1+ monocytes, which inhibit tumor-specific $\mathrm{T}$ cells. Multiple cell types have been shown to regulate tumor-specific responses.

Second, HSC transplants lead to at least two opposing activities that promote immunity and tolerance: activating naive $\mathrm{CD}^{+} \mathrm{T}$ cells through MHC class I and all T cells through costimulation; and activating regulatory cells that dampen the immune response. Lin $^{-} / \mathrm{c}-\mathrm{kit}^{+}$HSCs might have the ability to directly affect $\mathrm{T}$ cell homeostasis, or, perhaps more likely, HSCs rapidly differentiate into APCs that present tumor antigen (53-55). Only when the potential effects of regulation are removed do the positive effects of HSC transplants on antitumor immunity become apparent. With respect to naive $\mathrm{T}$ cells, it can be surmised that HSC-derived APCs - rather than HSCs per se - amplify tumor immunity by priming naive tumor-specific $\mathrm{CD}^{+} \mathrm{T}$ cells via $\mathrm{MHC}$ class I and costimulation. The relative role of MHC class I expression on resident host APCs versus transplanted HSCs was not examined in the study under discussion. With respect to effector T cells, HSC-derived APCs provide costimulation and promote expansion of tumor-specific effector T cells, 
independent of MHC class I and independent of host T cells. Although in some models, expansion of transferred, experienced $\mathrm{T}$ cells is APC independent and IL-7 and IL-15 dependent, Zaft et al. (56) have shown that it can be dependent on resident CD $11 c^{+}$DCs. Thus, HSC-derived CD $11 c^{+}$DCs or other APCs might affect homeostasis of tumorspecific effector T cells. Whether costimulation is provided through soluble factors, as Wrzesinski and colleagues speculate (13), or cell surface molecules such as CD40, CD80, CD86, or CD134 ligand also remains to be elucidated. With respect to regulatory cells, HSC-derived APCs provide survival signals to residual radioresistant regulatory cells, including Tregs and NKT cells.

Third, the authors' supplemental data suggest that nonablative irradiation plus vaccination appears to be as effective as ablative irradiation and HSC transplantation: what then is the benefit of employing the more aggressive preparative regimen? On the face of it, the benefit could lie in cases where a suitable vaccine is not available to stimulate/activate the transferred $\mathrm{T}$ cells. In this scenario, it is likely that melanoma-reactive $\mathrm{CD}^{+} \mathrm{T}$ cells can still be generated from resected tumors (i.e., TILs), activated, and expanded ex vivo for adoptive transfer into hosts following myeloablation and HSC rescue (57).

Fourth, how generalizable are the findings? Although the transgenic mouse model in which the pmel-1 TCR is expressed-with the very high frequency (>90\%) of murine gp100 melanoma-associated antigen-reactive $\mathrm{CD}^{+} \mathrm{T}$ cells - is attractive from the standpoint of assay sensitivity/specificity as well its ability to tease out the operative mechanisms involved, it is unknown whether the added benefit of myeloablation and HSC transplant can be replicated in a more standard model, e.g., in conventional C57BL/6 mice undergoing lethal TBI and syngeneic HSC transplantation followed by the adoptive transfer of TILs containing a polyclonal population of tumor-specific $\mathrm{CD}^{+}$and $\mathrm{CD}^{+} \mathrm{T}$ cells. The latter would be more akin to the ongoing clinical trial in melanoma patients (30). Also of importance, earlier clinical studies involving the adoptive transfer of cloned $\mathrm{CD}^{+} \mathrm{T}$ cells did not result in any objective responses or any persistence of the transferred cells (including in the setting of nonmyeloablative conditioning) (31).

While it will be important to define the exact mechanism whereby HSCs can promote the expansion of adoptively trans- ferred antitumor $\mathrm{CD}^{+} \mathrm{T}$ cells, perhaps more important will be to put these exciting findings to the test in the clinic. Given the initial promising clinical results to date of the adoptive transfer of TILs (30) and TCR gene-modified peripheral blood lymphocytes in nonmyeloablated, lymphopenic melanoma patients (58), the stage is now set to carry the strategy further in the setting of myeloablation with HSC transplant rescue.

Address correspondence to: James J. Mulé, H. Lee Moffitt Cancer Center and Research Institute, University of South Florida, 12902 Magnolia Drive, SRB-3, Tampa, Florida 33612, USA. Phone: (813) 7451536; Fax: (813) 745-6188; E-mail:mulejj@ moffitt.usf.edu.

1. Lizee, G., Radvanyi, L.G., Overwijk, W.W., and Hwu, P. 2006. Improving antitumor immune responses by circumventing immunoregulatory cells and mechanisms. Clin. Cancer Res. 12:4794-4803.

2. Finke, J.H., et al. 1993. Loss of T-cell receptor zeta chain and p56lck in T-cells infiltrating human renal cell carcinoma. Cancer Res. 53:5613-5616.

3. Wang, L.X., et al. 2005. Interleukin-7-dependent expansion and persistence of melanoma-specific $\mathrm{T}$ cells in lymphodepleted mice lead to tumor regression and editing. Cancer Res. 65:10569-10577.

4. Gattinoni, L., et al. 2005. Removal of homeostatic cytokine sinks by lymphodepletion enhances the efficacy of adoptively transferred tumor-specific CD8+ T cells. J. Exp. Med. 202:907-912.

5. Mulé, J.J., Jones, F.R., Hellstrom, I., and Hellstrom, K.E. 1979. Selective localization of radiolabeled immune lymphocytes into syngeneic tumors. J. Immunol. 123:600-606.

6. Lugade, A.A., et al. 2005. Local radiation therapy of B16 melanoma tumors increases the generation of tumor antigen-specific effector cells that traffic to the tumor. J. Immunol. 174:7516-7523.

7. Wang, L.X., Shu, S., and Plautz, G.E. 2005. Host lymphodepletion augments $\mathrm{T}$ cell adoptive immunotherapy through enhanced intratumoral proliferation of effector cells. Cancer Res. 65:9547-9554.

8. Dummer, W., et al. 2002. T cell homeostatic proliferation elicits effective antitumor autoimmunity. J. Clin. Invest. 110:185-192. doi:10.1172/ JCI200215175.

9. Cho, B.K., Rao, V.P., Ge, Q., Eisen, H.N., and Chen, J. 2000. Homeostasis-stimulated proliferation drives naive $\mathrm{T}$ cells to differentiate directly into memory T cells. J. Exp. Med. 192:549-556.

10. Petrus, M.J., Williams, J.F., Eckhaus, M.A., Gress, R.E., and Fowler, D.H. 2000. An immunoablative regimen of fludarabine and cyclophosphamide prevents fully MHC-mismatched murine marrow graft rejection independent of GVHD. Biol. Blood Marrow Transplant. 6:182-189.

11. Ercolini, A.M., et al. 2005. Recruitment of latent pools of high-avidity CD8(+) T cells to the antitumor immune response. J. Exp. Med. 201:1591-1602.

12. Estin, C.D., Stevenson, U.S., Hellstrom, I., and Hellstrom, K.E. 1989. Cyclophosphamide potentiates the antitumor activity of v-p97NY. Cell. Immunol. 120:126-131.

13. Wrzesinski, C., et al. 2007. Hematopoietic stem cells promote the expansion and function of adoptively transferred antitumor $\mathrm{CD}^{+} \mathrm{T}$ cells. J. Clin. Invest. 117:492-501. doi:10.1172/JCI30414.

14. Nobusada, K., Kuhn, L., Pilon-Thomas, S.A., and Mulé, J.J. 2005. Adoptive immunotherapy com- bined with a dendritic cell-based vaccine results in the rejection of established tumor in a murine melanoma model. J. Immunother. 28:614.

15. Ma, J., et al. 2003. Anti-tumor T cell response and protective immunity in mice that received sublethal irradiation and immune reconstitution. Eur. J. Immunol. 33:2123-2132.

16. La Gruta, N.L., Driel, I.R., and Gleeson, P.A. 2000. Peripheral $\mathrm{T}$ cell expansion in lymphopenic mice results in a restricted $\mathrm{T}$ cell repertoire. Eur. J. Immunol. 12:3380-3386.

17. Ge, Q., Rao, V.P., Cho, B.K., Eisen, H.N., and Chen, J. 2001. Dependence of lymphopenia-induced T cell proliferation on the abundance of peptide/ MHC epitopes and strength of their interaction with T cell receptors. Proc. Natl. Acad. Sci. U. S. A. 98:1728-1733.

18. Hu, H.M., Poehlein, C.H., Urba, W.J., and Fox, B.A. 2002. Development of antitumor immune responses in reconstituted lymphopenic hosts. Cancer Res. 62:3914-3919.

19. Ge, Q., Palliser, D., Eisen, H.N., and Chen, J. 2002. Homeostatic $\mathrm{T}$ cell proliferation in a T cell-dendritic cell coculture system. Proc. Natl. Acad. Sci. U. S. A. 99:2983-2988.

20. Lou, Y., et al. 2004. Dendritic cells strongly boost the antitumor activity of adoptively transferred $\mathrm{T}$ cells in vivo. Cancer Res. 64:6783-6790.

21. Oehen, S., and Brduscha-Riem, K. 1999. Naive cytotoxic $\mathrm{T}$ lymphocytes spontaneously acquire effector function in lymphocytopenic recipients: a pitfall for T cell memory studies? Eur. J. Immunol. 29:608-614.

22. Kieper, W.C., and Jameson, S.C. 1999. Homeostatic expansion and phenotypic conversion of naive $\mathrm{T}$ cells in response to self peptide/MHC ligands. Proc. Natl. Acad. Sci. U. S. A. 96:13306-13311.

23. Goldrath, A.W., and Bevan, M.J. 1999. Low-affinity ligands for the TCR drive proliferation of mature $\mathrm{CD}^{+} \mathrm{T}$ cells in lymphopenic hosts. Immunity. 11:183-190.

24. Klebanoff, C.A., et al. 2005. Central memory self/ tumor-reactive $\mathrm{CD}^{+} \mathrm{T}$ cells confer superior antitumor immunity compared with effector memory T cells. Proc. Natl. Acad. Sci. U. S. A. 102:9571-9576.

25. Klebanoff, C.A., Gattinoni, L., and Restifo, N.P. 2006. CD8 ${ }^{+}$T-cell memory in tumor immunology and immunotherapy. Immunol. Rev. 211:214-224.

26. Badovinac, V.P., Messingham, K.A., Jabbari, A., Haring, J.S., and Harty, J.T. 2005. Accelerated CD8 ${ }^{+}$ T-cell memory and prime-boost response after dendritic-cell vaccination. Nat. Med. 11:748-756.

27. Sallusto, F., Lenig, D., Forster, R., Lipp, M., and Lanzavecchia, A. 1999. Two subsets of memory T lymphocytes with distinct homing potentials and effector functions. Nature. 401:708-712.

28. He, H., et al. 2006. Combined IL-21 and low-dose IL-2 therapy induces anti-tumor immunity and long-term curative effects in a murine melanoma tumor model. J. Transl. Med. 4:24.

29. Dudley, M.E., and Rosenberg, S.A. 2003. Adoptivecell-transfer therapy for the treatment of patients with cancer. Nat. Rev. Cancer. 3:666-675.

30. Dudley, M.E., et al. 2002. Cancer regression and autoimmunity in patients after clonal repopulation with antitumor lymphocytes. Science. 298:850-854.

31. Dudley, M.E., et al. 2002. A phase I study of nonmyeloablative chemotherapy and adoptive transfer of autologous tumor antigen-specific $\mathrm{T}$ lymphocytes in patients with metastatic melanoma. J. Immunother. 25:243-251.

32. Asavaroengchai, W., Kotera, Y., Koike, N., PilonThomas, S., and Mulé, J.J. 2004. Augmentation of antitumor immune responses after adoptive transfer of bone marrow derived from donors immunized with tumor lysate-pulsed dendritic cells. Biol. Blood Marrow Transplant. 10:524-533.

33. Bacci, A., et al. 2002. Dendritic cells pulsed with 
fungal RNA induce protective immunity to Candida albicans in hematopoietic transplantation. J. Immunol. 168:2904-2913.

34. Asavaroengchai, W., Kotera, Y., and Mulé, J.J. 2002. Tumor lysate-pulsed dendritic cells can elicit an effective antitumor immune response during early lymphoid recovery. Proc. Natl. Acad. Sci. U. S. A. 99:931-936

35. Moyer, J.S., Maine, G., and Mulé, J.J. 2006. Early vaccination with tumor lysate-pulsed dendritic cells after allogeneic bone marrow transplantation has antitumor effects. Biol. Blood Marrow Transplant. 12:1010-1019.

36. Ge, Q., Hu, H., Eisen, H.N., and Chen, J. 2002. Different contributions of thymopoiesis and homeostasis-driven proliferation to the reconstitution of naive and memory T cell compartments. Proc. Natl. Acad. Sci. U. S. A. 99:2989-2994.

37. Mazo, I.B., et al. 2005. Bone marrow is a major reservoir and site of recruitment for central memory $\mathrm{CD}^{+} \mathrm{T}$ cells. Immunity. 22:259-270

38. Leen, A.M., et al. 2005. T-cell immunotherapy for adenoviral infections of stem-cell transplant recipients. Ann. N. Y. Acad. Sci. 1062:104-115.

39. Bollard, C.M., et al. 2006. In vivo expansion of LMP 1 - and 2 -specific T-cells in a patient who received donor-derived EBV-specific T-cells after allogeneic stem cell transplantation. Leuk. Lymphoma. 47:837-842.

40. Leen, A.M., et al. 2006. Monoculture-derived T lymphocytes specific for multiple viruses expand and produce clinically relevant effects in immunocom- promised individuals. Nat. Med. 12:1160-1166.

41. Riddell, S.R., Bleakley, M., Nishida, T., Berger, C. and Warren, E.H. 2006. Adoptive transfer of allogeneic antigen-specific T cells. Biol. Blood Marrow Transplant. 12:9-12.

42. Riddell, S.R., and Greenberg, P.D. 1997. T cell therapy of human CMV and EBV infection in immunocompromised hosts. Rev. Med. Virol. 7:181-192.

43. Kwak, L.W., Neelapu, S.S., and Bishop, M.R. 2004 Adoptive immunotherapy with antigen-specific $\mathrm{T}$ cells in myeloma: a model of tumor-specific donor lymphocyte infusion. Semin. Oncol. 31:37-46.

44. Neelapu, S.S., et al. 2005. Tumor antigen immunization of sibling stem cell transplant donors in multiple myeloma. Bone Marrow Transplant. 36:315-323

45. Rapoport, A.P., et al. 2005. Restoration of immunity in lymphopenic individuals with cancer by vaccination and adoptive T-cell transfer. Nat. Med. 11:1230-1237.

46. Teague, R.M., et al. 2006. Interleukin-15 rescues tolerant $\mathrm{CD}^{+} \mathrm{T}$ cells for use in adoptive immunotherapy of established tumors. Nat. Med. 12:335-341.

47. Tuma, R.A., Giannino, R., Guirnalda, P., Leinr, I., and Pamer, E.G. 2002. Rescue of CD8 T cellmediated antimicrobial immunity with a nonspecific inflammatory stimulus. J. Clin. Invest. 100:1493-1501.

48. Farrell, A. 2006. Defeating T-cell fatigue in HIV. Nat. Med. 12:1124-1125.

49. Overwijk, W.W., et al. 2003. Tumor regression and autoimmunity after reversal of a functionally toler- ant state of self-reactive CD8+ T cells. J. Exp. Med. 198:569-580.

50. Collin, M.P., et al. 2006. The fate of human Langerhans cells in hematopoietic stem cell transplantation. J. Exp. Med. 203:27-33.

51. Matte, C.C., et al. 2004. Donor APCs are required for maximal GVHD but not for GVL. Nat. Med. 10:987-992.

52. Reddy, P., et al. 2005. A crucial role for antigen-presenting cells and alloantigen expression in graft-versus-leukemia responses. Nat. Med. 11:1244-1249.

53. Feng, B., et al. 2000. Development of mouse dendritic cells from lineage-negative c-kit(low) pluripotent hemopoietic stem cells in vitro. Stem Cells. 18:53-60.

54. Ginhoux, F., et al. 2006. Langerhans cells arise from monocytes in vivo. Nat. Immunol. 7:265-273.

55. Merad, M., et al. 2004. Depletion of host Langerhans cells before transplantation of donor alloreactive $\mathrm{T}$ cells prevents skin graft-versus-host disease. Nat. Med. 10:510-517.

56. Zaft, T., Sapoznikov, A., Krauthgamer, R., Littman, D.R., and Jung, S. 2005. CD11c-high dendritic cell ablation impairs lymphopenia-driven proliferation of naive and memory $\mathrm{CD}^{+} \mathrm{T}$ cells. J. Immunol. 175:6428-6435.

57. Gattinoni, L., Powell, D.J., Rosenberg, S.A., and Restifo, N.P. 2006. Adoptive immunotherapy of cancer: building on success. Nat. Rev. Immunol. 6:383-393.

58. Morgan, R.A., et al. 2006. Cancer regression in patients after transfer of genetically engineered lymphocytes. Science. 314:126-129.

\title{
A link between protein translation and body weight
}

\section{Liangyou Rui}

\author{
Department of Molecular and Integrative Physiology, University of Michigan Medical School, Ann Arbor, Michigan, USA.
}

\begin{abstract}
Nutrient overload induces obesity, a primary risk factor for type 2 diabetes. Ribosomal biogenesis and protein synthesis, which are controlled by the mammalian target of rapamycin (mTOR), are primary energy-consuming processes in cells. mTOR phosphorylates and inactivates members of the eukaryotic translation initiation factor 4E-binding (eIF4E-binding) protein (4E-BP) family, which are translational repressors of $5^{\prime}$ cap-dependent protein synthesis. In this issue of the JCI, Le Bacquer et al. report that simultaneous deletion of both 4E-BP1 and 4E-BP2 in mice results in insulin resistance, decreased energy expenditure, and increased adipogenesis (see the related article beginning on page 387 ). These findings link protein synthesis, insulin sensitivity, and body weight.
\end{abstract}

Food (energy) shortage is a constant threat to the survival of a species. Individuals who can efficiently maintain their body weight via energy conservation have an increased

Nonstandard abbreviations used: DKO, double knockout; 4E-BP, eIF4E-binding protein; eIF, eukaryotic translation initiation factor; IRS-1, insulin receptor substrate 1 ; mTOR, mammalian target of rapamycin; mTORC, mTOR complex; PDK1, phosphoinositidedependent protein kinase 1; S6K, ribosomal protein S6 kinase; $5^{\prime}$ UTR, $5^{\prime}$ untranslated region.

Conflict of interest: The author has declared that no conflict of interest exists.

Citation for this article: J. Clin. Invest. 117:310-313 (2007). doi:10.1172/JCI31289. chance of survival and propagation during times when food supply is limited. Nutrients and hormones activate multiple evolutionarily conserved signaling pathways that govern the balance between energy intake and expenditure. Mammalian target of rapamycin (mTOR) is a well-conserved serine/threonine protein kinase that functions as an intracellular nutrient sensor to control protein synthesis, cell growth, and metabolism. In this issue of the JCI, Le Bacquer et al. demonstrate that the eukaryotic translation initiation factor 4E-binding (eIF4E-binding) protein (4E-BP) family of translational repressors, which are physiologic substrates of mTOR, play a key role in regulating body weight and glucose homeostasis in mice (1).

\section{mTOR regulation of energy and glucose metabolism}

mTOR is a member of the phosphoinositide kinase-related kinase family and is activated by nutrients (e.g., branched-chain amino acids) as well as by metabolic hormones, growth factors, and cytokines. mTOR binds to other regulatory components to form 2 distinct multiprotein complexes. The first complex, mTORC1, contains mTOR, regulator-associated protein of mTOR (Raptor), and $G$ protein $\beta$ subunit-like protein $(\mathrm{G} \beta \mathrm{L})$. The second complex, mTORC2, contains mTOR, rapamycin-insensitive companion of mTOR (Rictor), mammalian stress-activated protein kinase-interacting protein $1(\mathrm{mSin} 1)$, and $\mathrm{G} \beta \mathrm{L}$ (Figure 1). The adaptor proteins Raptor and Rictor determine the substrate specificity of mTORC1 and mTORC2, respectively. mTORC1 specifically phosphorylates ribosomal protein 